\title{
Power Load Studies in JET and ASDEX Upgrade with full-W divertors
}

\author{
B Sieglin ${ }^{1}$, T Eich $^{1}$, A Scarabosio ${ }^{1}$, G Arnoux ${ }^{2}$, I Balboa ${ }^{2}$, S \\ Devaux $^{1}$, A Herrmann ${ }^{1}$, A Hoppe ${ }^{1}$, M Hölzl $^{1}$ A Kallenbach ${ }^{1}, \mathbf{P}$ \\ Lang ${ }^{1}$, G F Matthews ${ }^{2}$, S Marsen ${ }^{3}$, S Pamela ${ }^{2}$, M Rack ${ }^{4}$, R \\ Wenninger $^{1}$, the ASDEX Upgrade Team and JET EFDA \\ Contributors $\ddagger$ \\ JET-EFDA, Culham Science Centre, Abingdon, OX14 3DB, United Kingdom \\ ${ }^{1}$ Max-Planck-Institute for Plasma Physics, Boltzmannstr. 2, D-85748 Garching, \\ Germany \\ ${ }^{2}$ CCFE, EURATOM-Association, Culham Science Centre, Abingdon, OX14 3DB, \\ United Kingdom \\ 3 Max-Planck-Institute for Plasma Physics, Wendelsteinstr.1, 17491 Greifswald, \\ Germany \\ ${ }^{4}$ Institute of Energy and Climate Research - Plasma Physics, Forschungszentrum \\ Jülich GmbH, Association EURATOM-FZJ, Partner in the Trilateral Euregio \\ Cluster, D-52425 Jülich, Germany \\ E-mail: Bernhard.Sieglin@ipp.mpg.de
}

\begin{abstract}
For the design and the operation of large fusion devices a detailed understanding of the power exhaust processes is necessary. This paper will give an overview of the current research on the divertor power load mechanisms. The results shown are obtained in JET with the ITER like wall and ASDEX Upgrade with tungsten coated plasma facing components. The challenges of infrared thermography on an ITER like bulk tungsten divertor are presented. For the steady state heat load, the power fall-off length $\lambda_{q}$ in JET-ILW is compared to an empirical scaling found in JET and ASDEX Upgrade with carbon plasma facing components. A first attempt to scale the divertor broadening $S$ in ASDEX Upgrade with tungsten plasma facing components is shown. The ELM duration $t_{E L M}$ in JET-C and JET-ILW is compared. For similar pedestal conditions $\left(T_{e, p e d}\right.$ and $\left.n_{e, p e d}\right)$, similar ELM durations are found in JET-C and JET-ILW. For higher $n_{e, p e d}$ at the same pedestal pressure $p_{e, p e d}$, longer ELM durations are found in JET-ILW. The pedestal pressure $p_{e, p e d}$ is found to be a good qualifier for the ELM energy fluency in both JET-C and JET-ILW. Improved diagnostic capabilities reveal ELM substructures on the divertor target occurring a few milliseconds before the ELM crash.
\end{abstract}

$\ddagger$ See the Appendix of F.Romanelli et al., Proceedings of the 24th IAEA Fusion Energy Conference 2012, San Diego, USA 


\section{Introduction}

Control of power exhaust in the divertor is a critical topic for present day devices (e.g. ASDEX Upgrade and JET) and even more for future tokamak devices such as ITER where significantly more power is entering the scrape-off layer. Quantification and interpretation of the power and energy exhaust at the divertor target plates in these present-day devices in the so-called base line scenario, type I ELMy H-mode, is crucial for the extrapolation to ITER. The power handling of the involved target plates is getting more critcial as plasma-facing components made of carbon-based materials have been in recent years replaced by full metallic components made of tungsten. ASDEX Upgrade demonstrated operation with a full tungsten wall in 2007. Since 2011, JET is equipped with the ITER-Like Wall [1], with plasma facing components (PFC) made of beryllium in the main chamber and tungsten in the divertor. The exchange of the wall material was in the first place driven by the in acceptable high tritium retention in the presence of carbon-based PFCs. Both ASDEX-Upgrade and JET-ILW demonstrated the reduction of tritium retention to acceptable levels $[2,3]$.

\section{Challenges of Heat Flux Measurements on W PFCs}

For typical divertor target temperatures between $300-1500^{\circ} \mathrm{C}$ bulk tungsten has a low emissivity of about $\epsilon=0.05-0.2$ at the wavelength range of $4.0-4.5 \mu \mathrm{m}$ used for the IR measurement $[4,5]$. This low emissivity leads to a low photon flux at low temperatures, where reflected light from the main chamber can influence the IR measurement. For high temperatures, connected to regions with high heat load as observed near the strike line and during ELMs, no significant influence $(<5 \%)$ of reflections has been estimated.

In contrast to the CFC target in JET-C, the bulk tungsten outer target plate in JET-ILW is not a uniform block, but consists of four stacks of 24 lamellae each [6]. This castellation poses a challenge on the IR measurements of the surface temperature distribution. The toroidal width of each lamella is about $6 \mathrm{~mm}$, with a gap of about $1 \mathrm{~mm}$ in between two neighboring lamellae. The JET IR system for the outer horizontal divertor target has a spatial resolution of about $1.7 \mathrm{~mm}[5]$. Therefore an exact positioning of the measurement has to be ensured throughout the whole plasma pulse, in order to avoid a corruption of the measured surface temperature due to the influence of the toroidal gap.

During plasma operation, magnetic forces induced by the poloidal field coils and the plasma current itself, cause a slight inclination (about $1 \mathrm{~cm}$ related to the target) of the mounting of the camera on top of the JET device. The impact of the camera movement on the measurement on the target plate has been corrected by two techniques. The first technique is using a phase correlation method to calculate the movement of the camera directly from the measured data. For the second technique the movement in different test pulses with and without plasma and different poloidal field currents were analysed using the first technique. The results were used to calculate the movement based on 
the plasma and various coil currents. This method has been validated using discharges with different plasma scenarios, where the IR measurement allowed the application of the first technique. An accuracy of about $0.5 \mathrm{~mm}(\sim 1 / 4$ pixel $)$ is reached. Theses techniques were used for the IR measurements during the 2011-2012 campaign in the JET-ILW. For castellated structures, as foreseen in ITER, those methods are thought to be a valuable tool for IR thermography.

\section{Dedicated Discharges}

To obtain optimal IR measurements, dedicated discharges have been conducted in JETILW.

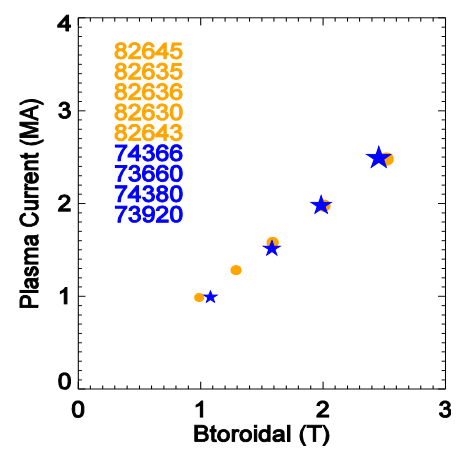

(a) Magnetic field and plasma current.

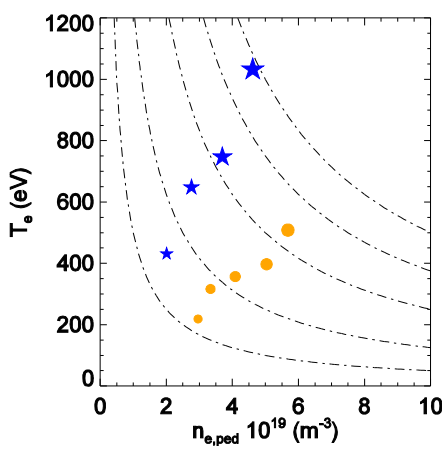

(b) Pedestal electron temperature and density.

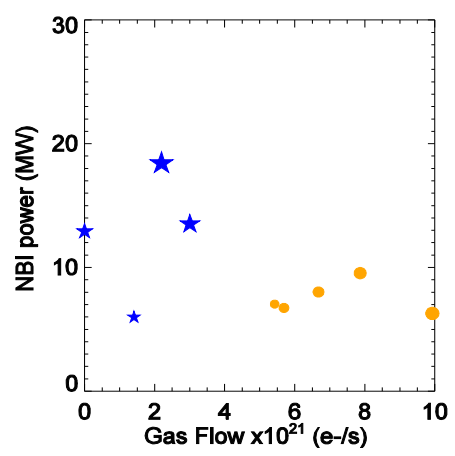

(c) Heating power and gas fueling rate.

Figure 1. Comparison between dedicated discharges in JET-C (blue) and JET-ILW with low heating power (orange).

Figure 1 shows the obtained plasma conditions, for a scan in magnetic field $B_{t}$ and plasma current $I_{p}$ in JET-ILW with low heating power $P_{\text {heat }}<10 \mathrm{MW}$. The reference discharges from JET-C are shown in blue. JET-ILW was operated at a higher gas flow $\left(\Gamma_{D}=5-10 \cdot 10^{21} \mathrm{~s}^{-1}\right)$ compared to JET-C $\left(\Gamma_{D}=0-3 \cdot 10^{21} \mathrm{~s}^{-1}\right)$ to prevent impurity accumulation $[7,8]$ (Figure 1(c)). The higher gas flux and the slightly lower heating power in JET-ILW lead to a different pedestal composition [9]. The achieved pedestal electron density is higher and the pedestal electron temperature is lower (Figure 1(b)). Comparable discharges were carried out at the last phase of JET-C operation with the outer target plate of the same geometry made by CFC.

In a second attempt discharges at higher auxiliary neutral beam heating power of up to $26 \mathrm{MW}$ have been executed in order to recover the pedestal temperatures observed in JET-C with the JET-ILW (Figure 2(c)). For these discharges similar pedestal conditions as in JET-C have been reached (Figure 2(b)). 


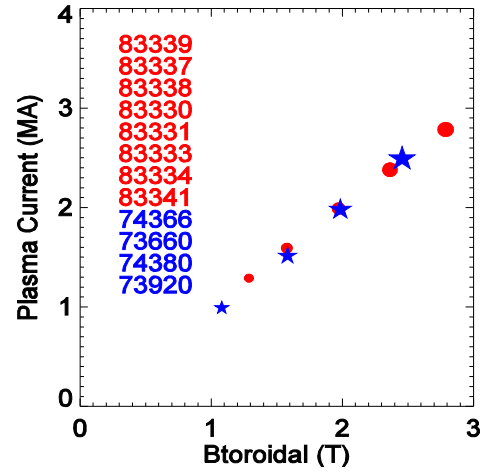

(a) Magnetic field and plasma current.

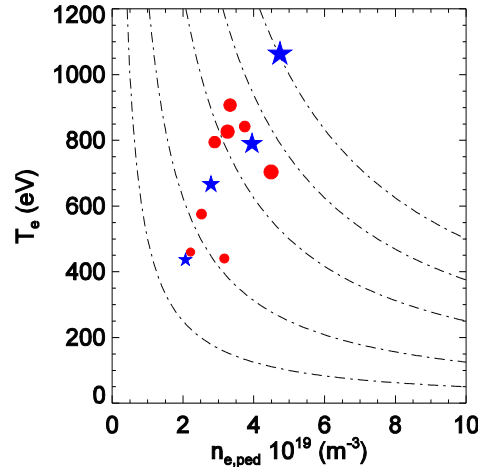

(b) Pedestal electron temperature and density.

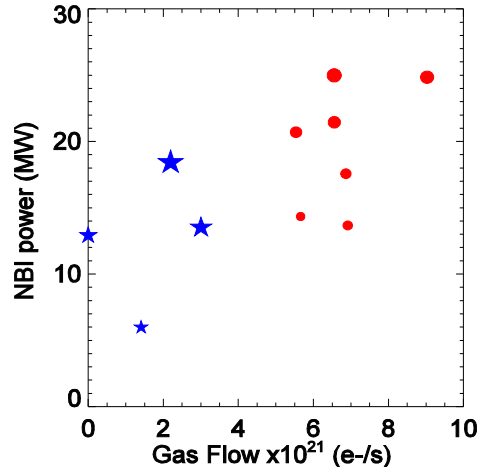

(c) Heating power and gas fueling rate.

Figure 2. Comparison between dedicated discharges in JET-C (blue) and JET-ILW with high heating power (red).

\section{Steady State Heat Load}

In this section the steady-state heat flux pattern at the outer divertor target is characterised. This applies to L-mode and inter-ELM phases in H-mode. For the target heat flux distribution a model was presented in [10].

$$
q(\bar{s})=\frac{q_{0}}{2} \exp \left(\left(\frac{S}{2 \lambda_{q}}\right)^{2}-\frac{\bar{s}}{\lambda_{q} f_{x}}\right) \operatorname{erfc}\left(\frac{S}{2 \lambda_{q}}-\frac{\bar{s}}{S f_{x}}\right)
$$

Where $\lambda_{q}$ is the power fall-off length, $S$ the divertor broadening, $\bar{s}$ the target position relative to the strike line, $q_{0}$ the peak heat flux density at the divertor entrance and $f_{x}$ the flux expansion for the given magnetic configuration.

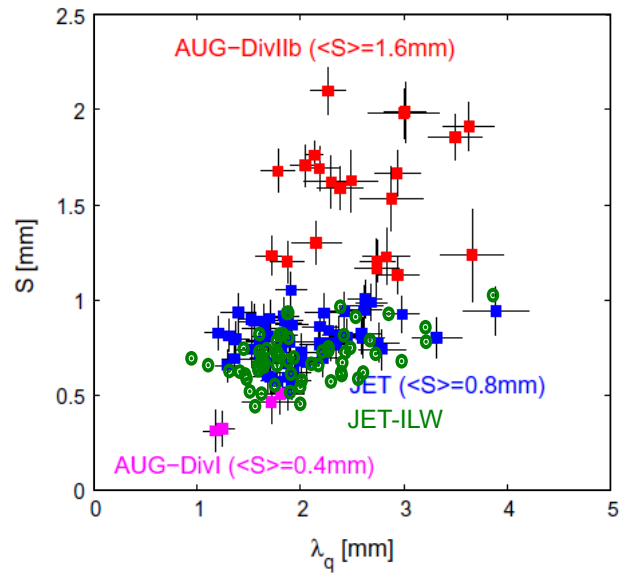

Figure 3. Divertor broadening $\mathrm{S}$ and power fall-off length in H-Mode for ASDEX Upgrade (Divertor I (magenta) and divertor IIb (red)), JET-C (blue) and JET-ILW (green). 
Figure 3 shows the measured divertor broadening and power fall-off length for ASDEX Upgrade (Divertor I and IIb $[11,12]$ ) with carbon PFCs, JET-C and JETILW [13]. For the dedicated discharges, no difference in the steady state heat load on the target plates is observed, when comparing JET-C and JET-ILW. The data from ASDEX Upgrade shows a large difference in the measured divertor broadening $S$, dependent on the divertor configuration. Divertor I, which is an open configuration, has an average value of $S=0.4 \mathrm{~mm}$ and the closed divertor IIb has an $S$ of about $1.6 \mathrm{~mm}$.

\subsection{Power Fall Off-Length $\lambda_{q}$}

As a first step it was attempted to validate the empirical inter-ELM H-Mode scaling for the power fall-off length $\lambda_{q}$ (Equation (2)) [13], resulting from JET and ASDEX Upgrade data with carbon plasma facing components, in the JET-ILW.

$$
\lambda_{q, s c a l}[\mathrm{~mm}]=0.73 B_{t}^{-0.8} q_{c y l}^{1.2} P_{S O L}^{0.1} R^{0.0}
$$

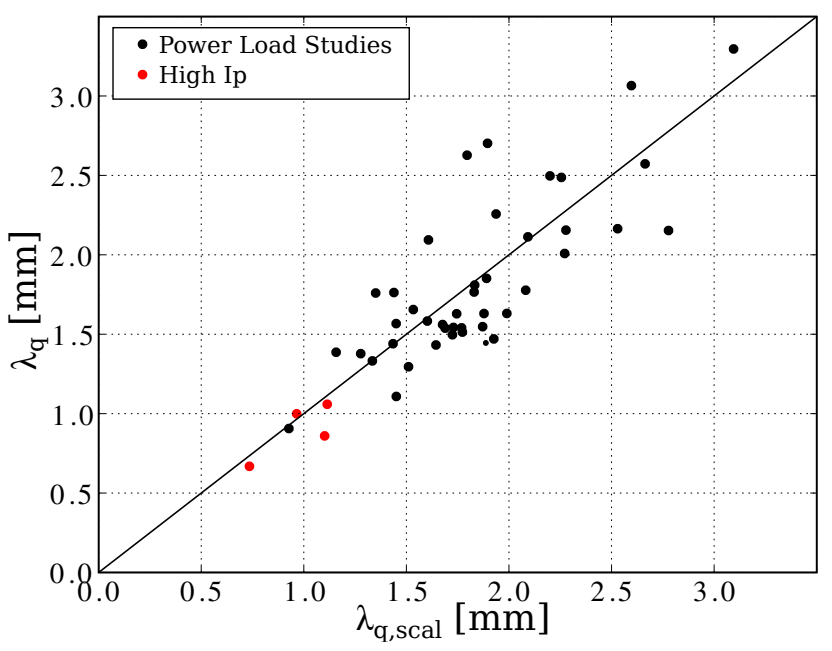

Figure 4. Comparison of the measured power fall-off length $\lambda_{q}$ in JET-ILW with the empirical scaling obtained in JET and ASDEX Upgrade with carbon plasma facing components (Equation (2)).

Figure 4 shows the measured power fall-off length $\lambda_{q}$ in JET-ILW compared to the empirical scaling in different operational regimes up to a toroidal magnetic field of $3.5 \mathrm{~T}$ and a plasma current of 3.5 MA. It is found, that the power fall-off length in JET-ILW for attached divertor conditions, can be described by the empirical scaling (2) found in carbon ASDEX Upgrade and JET-C. 


\subsection{Divertor Broadening $S$}

One important quantity for the assessment of the divertor power load in a tokamak is the integral power fall-off length $\lambda_{\text {int }}$ :

$$
\lambda_{\text {int }}=\frac{1}{q_{\max }} \int q(s) d s
$$

Where $q_{\max }$ is the peak heat flux on the target.

Using (1) a simple relation between $\lambda_{\text {int }}$ and both the power fall-off length $\lambda_{q}$ and the divertor broadening $S$ can be derived as shown by M.Makowski [14]:

$$
\lambda_{\text {int }} \approx \lambda_{q}+1.64 S
$$

With this it becomes clear that an understanding of the divertor broadening $S$ is mandatory to be able to estimate $\lambda_{\text {int }}$. For large values, $S$ can be the dominant contribution to $\lambda_{\text {int }}$, compensating for a small $\lambda_{q}$. In order to get well defined experimental and divertor conditions, dedicated L-Mode discharges with low recycling divertor conditions have been conducted in ASDEX Upgrade in deuterium and hydrogen. For the scaling of $S$, different quantities such as the pedestal electron density $n_{e, p e d}$, the

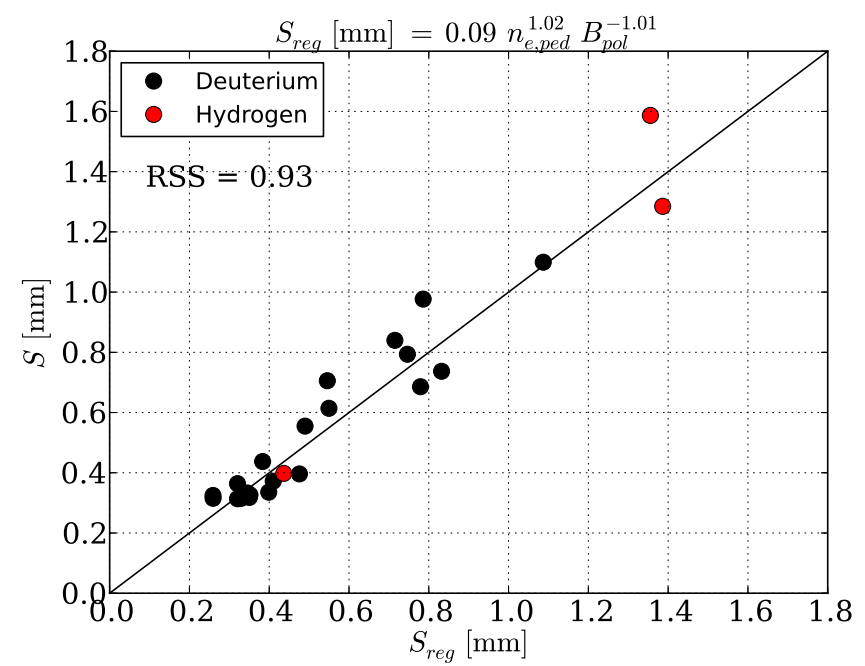

Figure 5. Regression results of the measured divertor broadening $S$ in ASDEX Upgrade L-Mode discharges.

neutral gas density in the divertor $n_{0, d i v}$, the specific isotope mass $A$ and the poloidal magnetic field $B_{p o l}$ have been examined. The best match of the measured divertor broadening, with a residual sum of squares of 0.93 , has been found with the following relation:

$$
S[\mathrm{~mm}] \widehat{=}(0.09 \pm 0.01) n_{e, p e d}^{1.02 \pm 0.03} B_{\text {pol }}^{-1.01 \pm 0.05}
$$

Where $n_{e, p e d}$ is the pedestal electron density in $10^{19} \mathrm{~m}^{-3}$ and $B_{\text {pol }}$ is the poloidal magnetic field in T:

$$
B_{p o l}=\frac{\mu_{0} I_{p}}{2 \pi a}\left(\frac{1+\kappa^{2}}{2}\right)^{-0.5}
$$


With the plasma current $I_{p}$, the minor radius $a$ and the elongation $\kappa$. So far no isotope dependence of the divertor broadening $S$ has been observed. It is to note here, that no dependence of $S$ on the neutral gas density $n_{0, d i v}$ has been found. For the investigation of the dependence of the divertor broadening on the machine size, the same study has to be performed in JET. This however is an ongoing process and can not be shown in this work.

\section{ELM Induced Transient Heat Load}

In H-Mode periodic edge-localised modes (ELM) induce high transient heat loads onto the divertor target plates. In contrast to the very localised steady state heat flux, the ELM heat load is unevenly deposited onto a comparable large area. For the measurement of the impact on the divertor target, the deposited energy fluency $\epsilon$ in $\mathrm{kJm}^{-2}$ is taken. In the dedicated discharges, the strike line position was set to ensure that the maximum of the energy fluency is within the observed area.

In devices with carbon plasma facing components the deposition of hydrocarbon layers can corrupt the measurements resulting in an overestimation of the peak heat flux [15]. In JET-ILW no formation of deposition layers (e.g. beryllium) has been observed which have an impact on the analysis on the outer horizontal target over the whole campaign.

For the estimation of divertor target life time, a important quantity is the heat impact factor $P \sqrt{t_{E L M}}[16]$ :

$$
P \sqrt{t_{E L M}}=\frac{\epsilon_{E L M}}{\sqrt{t_{E L M}}}=\frac{E_{E L M}}{A_{w e t}} \frac{1}{\sqrt{t_{c}}}
$$

Where $\epsilon_{E L M}$ is the deposited energy fluency and $t_{E L M}$ is the energy deposition time. For an acceptable life time of the ITER divertor, a limit of $500 \mathrm{kJm}^{-2}$ is found by material testing for a typical energy deposition time of $750 \mu s$ [16].

\subsection{ELM Duration $t_{E L M}$}

The first critical value for the impact of the ELM on the divertor target, is the ELM duration $t_{E L M}$ in which the ELM deposits its energy on the target. The pedestal electron temperatures and densities reached in JET-C (blue) and JET-ILW (red) are shown in figure 7(a) for the discharges used in the comparison. The distribution of the ELM duration found in JET-C and JET-ILW is shown in figure 6. In JET-C an average value of about $750 \mu \mathrm{s}$ is found and the variance in ELM duration is small. In the ILW, the average ELM duration is longer (about $2 \mathrm{~ms}$ ) and the distribution is wider. However the shortest ELMs reach the values observed in JET-C.

In figure 7(a) the electron temperature and densities are shown for a set of discharges in type I ELMy H-mode in JET-C and JET-ILW. JET-ILW operates typically at higher density for a given pedestal pressure in comparison with JET-C. As shown in figure 7(b) the ELM durations for the same pedestal conditions in JET-C (blue) and JET-ILW 


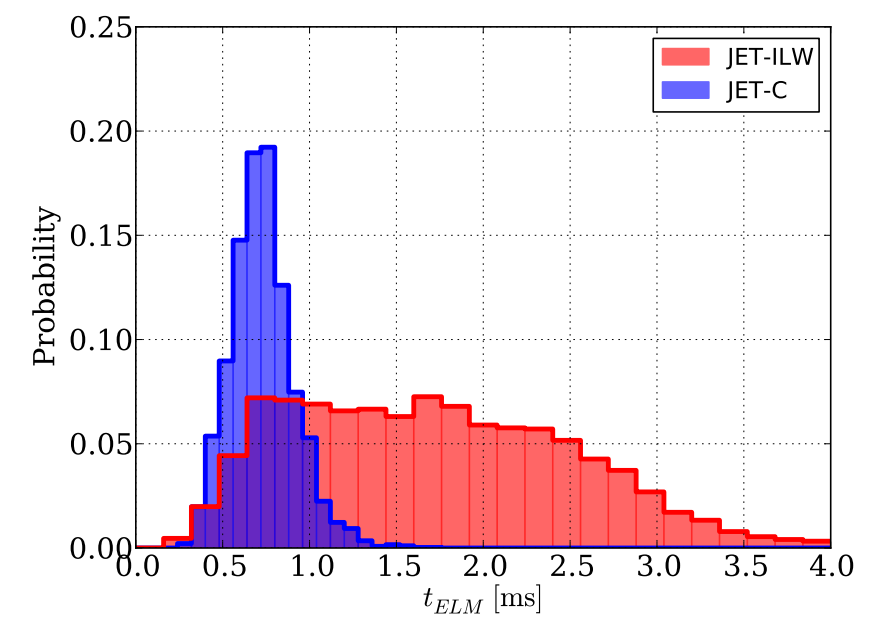

Figure 6. Comparison of the ELM duration $t_{E L M}$ for JET-C and JET-ILW for type I ELMy H-modes $\left(f_{E L M}<100 \mathrm{~Hz}\right)$.

(green) are comparable. For higher density at the same pedestal pressure (orange) in JET-ILW, the ELM duration becomes longer and the width of the distribution increases.

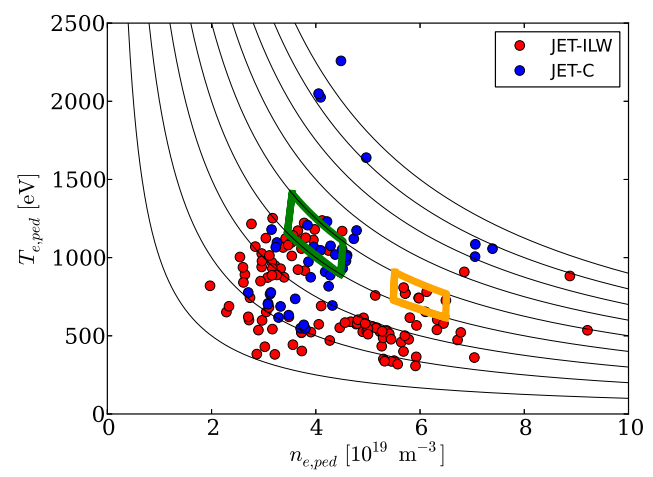

(a) Pedestal temperatures and densities for JET-C and JET-ILW with lines of constant pressure (black). Boxes show The operational space used for the comparison of the ELM duration for low (green) and high (density) at constant pedestal pressure..

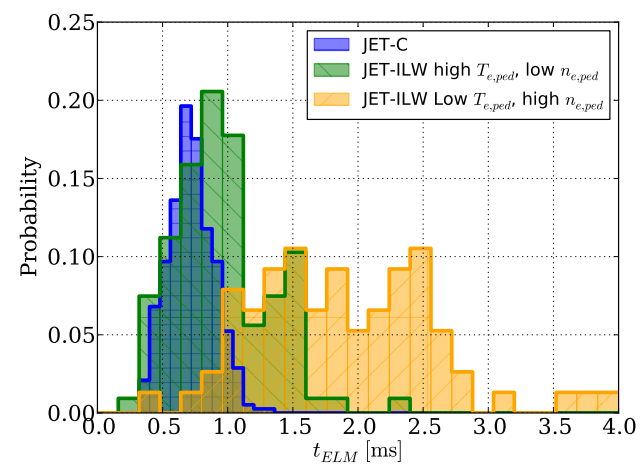

(b) ELM duration in JET-ILW at constant pressure but different pedestal composition $\left(n_{e, p e d} / T_{e, p e d}\right)$.

Figure 7. Comparison of ELM duration in ILW at different pedestal conditions.

This observation shows, that the ELM induced divertor heat load characteristic in JET-ILW is not largely different to JET-C. The operational space in which JET-ILW was operated has a high gas fueling than in JET-C, leading to longer ELM duration in the average. 


\subsection{ELM Energy Fluency $\epsilon_{\max }$}

In the following the ELM energy fluency in JET-ILW and JET-C are compared. The

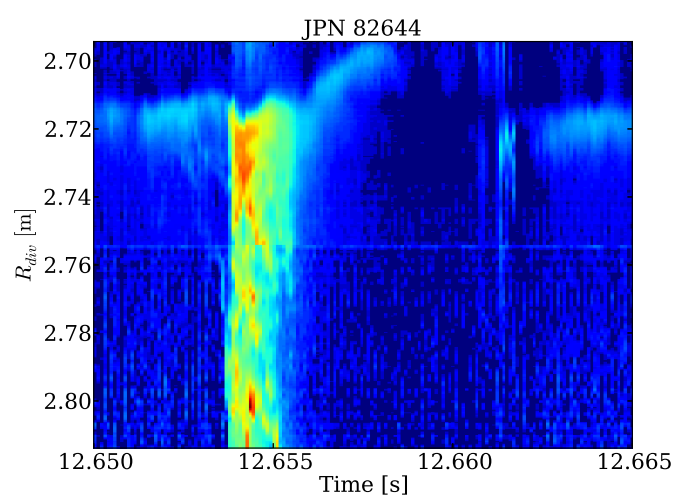

(a) ELM target heat load foot print in JETILW.

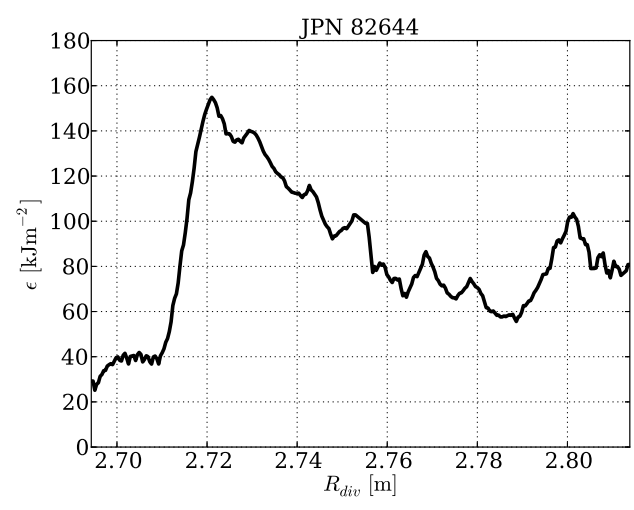

(b) Target ELM energy fluency profile.

Figure 8. Target heat load foot print and energy fluency for an Type-I ELM in JET-ILW.

energy fluency is calculated by integrating the heat flux $q(s, t)$ over the ELM duration:

$$
\epsilon(s)=\int_{E L M} q(s, t) d t
$$

Figure 8 shows an example of the ELM target heat load foot print and the resulting energy fluency profile. For the comparison of the ELM induced power load in JET-C and JET-ILW the peak energy fluency $\epsilon_{\max }$ is used.

$$
\epsilon_{\max }=\max (\epsilon(s))
$$

In order to account for the different magnetic inclination angles in JET-C and JETILW [17], the parallel energy fluency is calculated. Figure 9 shows the parallel peak energy fluency $\epsilon_{\|, \max }$ for JET-C and ILW plotted against the pedestal electron pressure $p_{e, p e d}$. It can be seen, that the energy fluency for both JET-C and JET-ILW is, within experimental scatter, the same for the same pedestal pressure.

\section{ELM Substructure}

An edge-localised mode (ELM) is understood as a magnetohydrodynamic (MHD) mode in the plasma edge that is identified as a peeling-ballooning mode [18]. Once an ELM becomes non-linearly unstable it causes an out-flux of particles from the plasma edge into the SOL[19]. This comes along with a strong drop of the electron pedestal temperature

and density. One part of the energy that leaves the confined plasma is radiated, another part is deposited as heat on the target plates in the divertor region. The temporal evolution of the heat deposition on the target plates is explained as a free-streaming process of the particles that are ejected from the confined plasma region into the SOL[20]. 


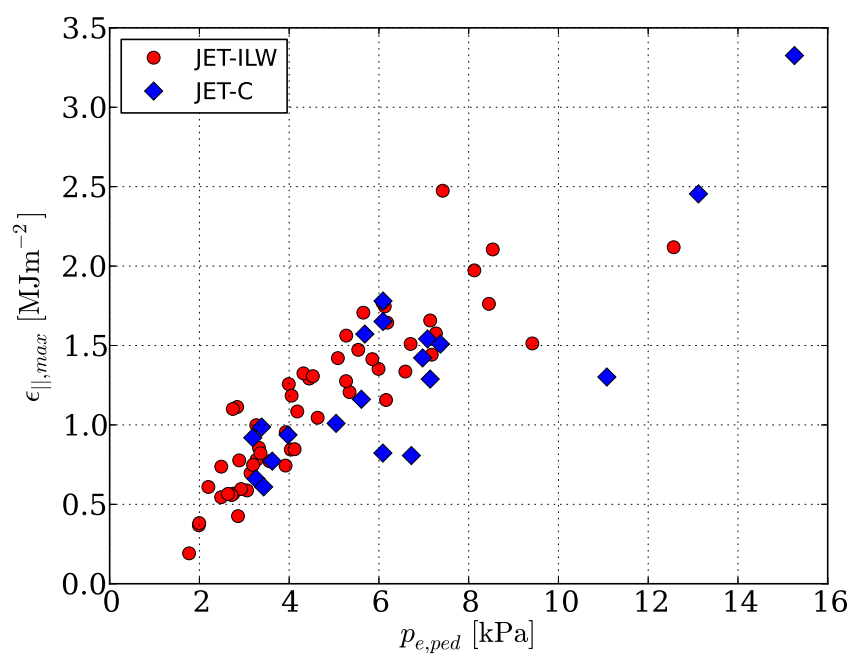

Figure 9. Parallel peak energy fluency $\epsilon_{\|, \text {max }}$ and pedestal electron pressure $p_{e, p e d}$ for JET-C (blue) and JET-ILW (red).

Typical rise times for the heat load on the target plates are in the order of a few hundred microseconds [4].

The heat load deposited during an ELM crash shows a characteristic pattern. An ELM causes a natural ergodisation of the plasma edge [21] which results in a random deposition of the heat load on the target plates, i.e. each ELM crash appears different. However, typical features have been observed, like a radial decay of the deposited energy on the target in the outward direction and filamentary structures that appear during the ELM crash. The number of the filamentary structures increases during the ELM crash and a quasi-toroidal mode number is defined for ELMs $[22,23]$. In the early phase of an ELM crash, a low mode number, $n=3$ to 5 , is identified, which increases to about $n=10$ to 20 during the ELM crash.

ELMs are associated with toroidally rotating filaments [24]. These filaments are created in the plasma edge and leave the confined region during the ELM crash. A radial propagation speed of the ELM filament in the range of $0.5 \mathrm{~km} \mathrm{~s}^{-1}$ to $2 \mathrm{~km} \mathrm{~s}^{-1}$ is found [25]. For a typical JET plasma shape, the radial spacing between wall and plasma is around $6-7 \mathrm{~cm}$ at the outer mid-plane. From this we calculate the time an ELM filament spends in the SOL before interacting with the wall and collapsing to be in the range of a hundred microseconds, which corresponds to the ELM crash rise time seen in the divertor heat load. The filamentary structures in the heat load have been linked to the appearance of ELM filaments and modeled with a reduced non-linear MHD code [26]. The modeling results show that these filamentary structures can be understood as poloidally rotating ballooning modes.

Recent experiments on JET-ILW [1] have shown the occurrence of radial propagating structures in the divertor heat load prior to the ELM crash, see figure 10. These pre-ELM structures have a life time of several milliseconds and can therefore not 


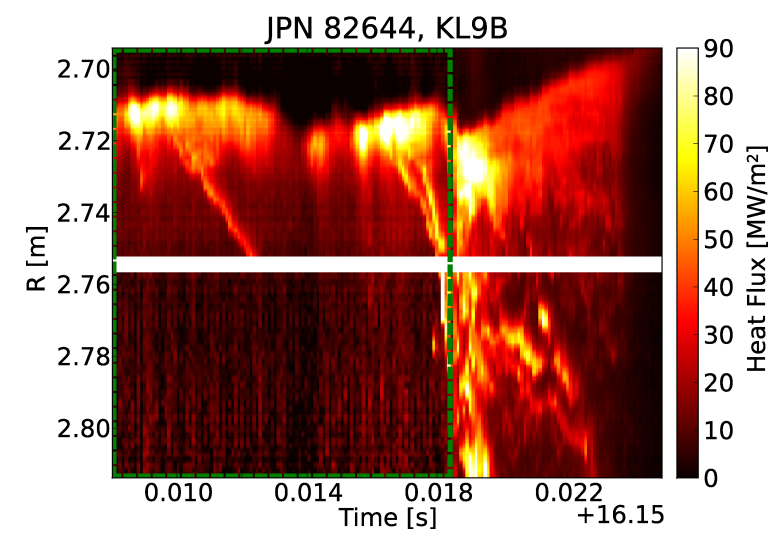

Figure 10. Heat load deposition pattern of one ELM for discharge with JET pulse number (JPN) 82644. The dashed rectangle marks a region where the heat load is magnified by a factor of five for an easier visibility of the pre-ELM structures.

be caused by the faster radial propagating ELM filaments that were discussed before. A few milliseconds before the ELM crash, a small increase of the heat flux on the original strike line position is seen. At that time the radial outward propagating structures are created at the same location. The pre-ELM structures propagate with a radial speed of around $10 \mathrm{~ms}^{-1}$ to $20 \mathrm{~ms}^{-1}$ and continue until the larger main ELM crash heat deposition pattern. The heat deposition pattern of the ELM crash appears to be influenced by the structures as stronger heat fluxes are measured at those positions where the structures connect to the pattern. After the ELM crash the structures vanish. The white line in figure 10 marks the gap between two stacks of the Tungsten divertor. Some pre-ELM structures seems to stop at that gap, see $8 \mathrm{~ms}$ before the ELM crash in figure 10. This either means that those pre-ELM structures do not necessary lead to an ELM crash, or that they do not reach the target plates because of the poloidal shadowing of the different stacks. In the given example, the very early pre-ELM structure is not seen on the outer stack ( $R \approx 2.757 \mathrm{~cm}$ to $2.814 \mathrm{~cm}$ ), but during the ELM crash an increased heat flux is seen at the position where this structure would have ended $(R \approx 2.81 \mathrm{~cm})$, if it continued with the same speed.

The pre-ELM structures are created before the ELM crash, i.e. before the large amount of energy and particles is lost into the SOL. Furthermore, the structures appear as several parallel stripes. This is similar to strike line splitting known from the effects of magnetic perturbations on low-confinement mode plasmas [27]. While ELM filaments cannot explain the observations, a possible explanation is an early change of the magnetic topology, several milliseconds before the ELM. This indicates that processes early in the ELM cycle lead to a perturbation of the plasma that finally influence the ELM crash heat deposition. During the ELM crash, the quasi-toroidal mode numbers are seen to increase from $n=3$ to 5 . The pre-ELM structures start with a single propagating stripe and increases in number until the ELM crash. The increase of stripes corresponds to an increasing quasi-toroidal mode number for the pre-ELM structures and links to the mode numbers during the ELM crash. The understanding of 
such an observation is of high interest in terms of ELM control. By knowing the physics of an ELM trigger the techniques for ELM control could be optimized.

\section{Summary}

In this paper an overview of current research activities of the divertor power load in JET and ASDEX Upgrade has been given. The challenges of IR thermography on castellated bulk tungsten have been discussed briefly. The main conclusions to be drawn from this paper are:

- Despite the change in the operational space in JET-ILW compared to JET-C, no change in the inter-ELM power fall-off length $\lambda_{q}$ and the divertor broadening $S$ has been observed.

- Using dedicated low recycling L-Mode discharges in ASDEX Upgrade, a scaling for the divertor broadening $S$ based on the pedestal top electron density $n_{e, p e d}$ and the poloidal magnetic field $B_{\text {pol }}$ has been found. Speculating on an absence of a size dependence in the scaling and taking values foreseen for ITER, the scaling delivers a divertor broadening $S$ for attached conditions, which lies in the same range as observed in ASDEX Upgrade and JET.

- In JET-ILW on average longer ELM durations (about $2 \mathrm{~ms}$ ) as compared to JET-C (about $750 \mu \mathrm{s}$ ) are found due to the on average higher pedestal densities.

- For both JET-ILW and JET-C, the pedestal electron pressure is found do order the parallel peak energy fluency $\epsilon_{\|, \max }$ deposited on the target. Thus within the experimental scatter no difference for $\epsilon_{\|, \text {max }}$ between JET-C and JET-ILW has been found.

- Independent on the on average longer ELM duration in JET-ILW, no difference in $\epsilon_{\|, \max }$ was observed.

- Filamentary pre-ELM structures have been observed on the outer divertor target several ms before the ELM crash, giving new input for the understanding of the physics triggering an ELM. Similar features have been observed in ASDEX Upgrade using magnetic probes[22].

In summary we note that tokamak operation in tungsten and CFC shows no significant difference in terms of divertor power load. Operation at higher pedestal density, as found in JET-ILW with higher gas flow when compared to JET-C, shows a beneficial effect due to the longer ELM time scale. Whether ITER with a tungsten divertor will have pedestal conditions which will foster longer ELMs is yet unclear. Further studies on the role of the pedestal conditions on the ELM duration need to be conducted in both JET and ASDEX Upgrade.

This work was supported by EURATOM and carried out within the framework of the European Fusion Development Agreement. The views and opinions expressed herein do not necessarily reflect those of the European Commission. 
[1] V. Philipps, Ph. Mertens, G.F. Matthews, and H. Maier. Overview of the jet iter-like wall project. Fusion Engineering and Design, 85(79):1581 - 1586, 2010.

[2] M. Mayer, V. Rohde, K. Sugiyama, J.L. Chen, X. Gong, C. Hopf, J. Likonen, S. Lindig, R. Neu, G. Ramos, E. Vainonen-Ahlgren, and A. Wiltner. Carbon balance and deuterium inventory from a carbon dominated to a full tungsten asdex upgrade. Journal of Nuclear Materials, 390 - 391(0):538 - 543, 2009.

[3] S. Brezinsek, S. Jachmich, M.F. Stamp, A.G. Meigs, J.W. Coenen, K. Krieger, C. Giroud, M. Groth, V. Philipps, S. Grn, R. Smith, G.J. van Rooij, D. Ivanova, and G.F. Matthews. Residual carbon content in the initial iter-like wall experiments at jet. Journal of Nuclear Materials, 438, Supplement(0):S303 - S308, 2013.

[4] T. Eich, H. Thomsen, W. Fundamenski, G. Arnoux, S. Brezinsek, S. Devaux, A. Herrmann, S. Jachmich, and J. Rapp. Type-i elm power deposition profile width and temporal shape in jet. Journal of Nuclear Materials, 415(1, Supplement):S856 - S859, 2011.

[5] I. Balboa, G. Arnoux, T. Eich, B. Sieglin, S. Devaux, W. Zeidner, C. Morlock, U. Kruezi, G. Sergienko, D. Kinna, P. D. Thomas, M. Rack, and JET EFDA Contributors. Upgrade of the infrared camera diagnostics for the jet iter-like wall divertor. Review of Scientific Instruments, 83(10):10D530, 2012.

[6] Ph. Mertens, T. Hirai, J. Linke, O. Neubauer, G. Pintsuk, V. Philipps, S. Sadakov, U. Samm, and B. Schweer. Conceptual design for a bulk tungsten divertor tile in jet. Fusion Engineering and Design, 82(1524):1833 - 1838, 2007.

[7] R. Neu, G. Arnoux, M. Beurskens, V. Bobkov, S. Brezinsek, J. Bucalossi, G. Calabro, C. Challis, J.W. Coenen, E. De La Luna, P.C. De Vries, R. Dux, L. Frassinetti, C. Giroud, M. Groth, J. Hobirk, E. Joffrin, P. Lang, M. Lehnen, E. Lerche, T. Loarer, P. Lomas, G. Maddison, C. Maggi, G. Matthews, S. Marsen, M.-L. Mayoral, A. Meigs, P. Mertens, I. Nunes, V. Philipps, T. Pch, F. Rimini, M. Sertoli, B. Sieglin, A.C.C. Sips, D. Van Eester, and G. Van Rooij. First operation with the jet international thermonuclear experimental reactor-like wall. Physics of Plasmas, 20(5), 2013.

[8] T. Puetterich, R. Dux, R. Neu, M. Bernert, M.N.A. Beurskens, V. Bobkov, S. Brezinsek, C. Challis, J.W. Coenen, A. Coffey, I. amd Czarnecka, C. Giroud, A. Gude, E. Joffrin, A. Kallenbach, M. Lehnen, E. Lerche, E. de la Luna, S. Marsen, G. Matthews, M-L. Mayoral, R.M. McDermott, A. Meigs, M Sertoli, G. van Rooij, J. Schweinzer, ASDEX Upgrade Team, and JET EFDA Contributors. Taming tungsten in jet and asdex upgrade. These proceedings., 2013.

[9] M.N.A. Beurskens, J. Schweinzer, C. Angioni, A. Burckhart, C.D. Challis, I. Chapman, R. Fischer, J. Flanagan, L. Frassinetti, C. Giroud, J. Hobirk, E. Joffrin, A. Kallenbach, M. Kempenaars, M. Leyland, P. Lomas, G. Maddison, M. Maslov, R. McDermott, R. Neu, I. Nunes, F. Ryter, S. Saarelma, P.A. Schneider, P. Snyder, G. Tardini, E. Viezzer, E. Wolfrum, the ASDEX Upgrade Team, and JET-EFDA Contributors. The effect of a metal wall on confinement in jet and asdex upgrade. These proceedings., 2013.

[10] T. Eich, B. Sieglin, A. Scarabosio, W. Fundamenski, R.J. Goldston, and A. Herrmann. Inter-elm power decay length for jet and asdex upgrade: Measurement and comparison with heuristic drift-based model. Physical Review Letters, 107(21), 2011.

[11] A Kallenbach, D Coster, J C Fuchs, H Y Guo, G Haas, A Herrmann, L D Horton, L C Ingesson, C F Maggi, G F Matthews, R D Monk, J Neuhauser, F Ryter, J Schweinzer, J Stober, W Suttrop, ASDEX Upgrade Team, and JET Team. Closed divertor operation in asdex upgrade and jet. Plasma Physics and Controlled Fusion, 41(12B):B177, 1999.

[12] R Neu, J C Fuchs, G Haas, A Herrmann, A Kallenbach, M Laux, J Neuhauser, F Ryter, J Gafert, O Gruber, M Kaufmann, B Kurzan, V Mertens, H W Mand V Rohde, A Sips, J Stober, B Streibl, W Treuterer, and the ASDEX Upgrade Team. Properties of the new divertor iib in asdex upgrade. Plasma Physics and Controlled Fusion, 44(6):1021, 2002.

[13] T. Eich, B. Sieglin, A. Scarabosio, A. Herrmann, A. Kallenbach, G.F. Matthews, S. Jachmich, S. Brezinsek, M. Rack, and R.J. Goldston. Empiricial scaling of inter-elm power widths in 
asdex upgrade and jet. Journal of Nuclear Materials, 2013.

[14] M.A. Makowski, D. Elder, T.K. Gray, B. Labombard, C.J. Lasnier, A.W. Leonard, R. Maingi, T.H. Osborne, P.C. Stangeby, J.L. Terry, and J. Watkins. Analysis of a multi-machine database on divertor heat fluxes. Physics of Plasmas, 19(5), 2012.

[15] S. Devaux, G. Arnoux, T. Eich, H. Thomsen, S. Brezinsek, P. Coad, J. Likonen, M. Stamp, and A. Widdowson. Surface layers effect on heat loads on the jet divertor targets. 38th EPS Conference on Plasma Physics 2011, EPS 2011 - Europhysics Conference Abstracts, 35 1:49-52, 2011.

[16] J. Linke, F. Escourbiac, I.V. Mazul, R. Nygren, M. R, J. Schlosser, and S. Suzuki. High heat flux testing of plasma facing materials and components - status and perspectives for iter related activities. Journal of Nuclear Materials, 367-370 B(SPEC. ISS.):1422-1431, 2007.

[17] S. Marsen, T. Eich, M. Groth, S. Jachmich, and B. Sieglin. Experimental sheath heat transmission factors in diverted plasmas in jet. Journal of Nuclear Materials, 438, Supplement(0):S393 - S396, 2013.

[18] J W Connor. Edge-localized modes - physics and theory. Plasma Physics and Controlled Fusion, 40(5):531, 1998.

[19] H. R. Wilson and S. C. Cowley. Theory for Explosive Ideal Magnetohydrodynamic Instabilities in Plasmas. Physical Review Letters, 92:175006, Apr 2004.

[20] T. Eich, A. Kallenbach, W. Fundamenski, A. Herrmann, and V. Naulin. On the asymmetries of elm divertor power deposition in jet and asdex upgrade. Journal of Nuclear Materials, 390391(0):760 - 763, 2009.

[21] T. Eich, A. Herrmann, and J. Neuhauser. Nonaxisymmetric Energy Deposition Pattern on ASDEX Upgrade Divertor Target Plates during Type-I Edge-Localized Modes. Physical Review Letters, 91:195003, Nov 2003.

[22] T. Eich, A. Herrmann, J. Neuhauser, R. Dux, J.C. Fuchs, S. Gnter, L.D. Horton, A. Kallenbach, P.T. Lang, C.F. Maggi, M. Maraschek, V. Rohde, and W. Schneider. Type-I ELM substructure on the divertor target plates in ASDEX Upgrade. Plasma Physics and Controlled Fusion, 47(6):815-842, 2005.

[23] S. Devaux, T. Eich, G. Arnoux, W. Fundamenski, and H. Thomsen. Type-i elm filamentary substructure on the jet divertor target. Journal of Nuclear Materials, 415(1, Supplement):S865 - S868, 2011.

[24] A. Kirk, B. Koch, R. Scannell, H. R. Wilson, G. Counsell, J. Dowling, A. Herrmann, R. Martin, and M. Walsh. Evolution of filament structures during edge-localized modes in the mast tokamak. Physical Review Letters, 96:185001, May 2006.

[25] A. Kirk, S. Lisgo, E. Nardon, T. Eich, A. Herrmann, A. Kallenbach, and A. Loarte. Physics of elm power fluxes to plasma facing components and implications for iter. Journal of Nuclear Materials, 390-391(0):727 - 732, 2009.

[26] S J P Pamela, G T A Huysmans, M N A Beurskens, S Devaux, T Eich, S Benkadda, and JET EFDA contributors. Nonlinear mhd simulations of edge-localized-modes in jet. Plasma Physics and Controlled Fusion, 53(5):054014, 2011.

[27] D.M. Harting, Y. Liang, S. Jachmich, R. Koslowski, G. Arnoux, S. Devaux, T. Eich, E. Nardon, D. Reiter, H. Thomsen, and JET EFDA contributors. Strike point splitting in the heat and particle flux profiles compared with the edge magnetic topology in a $\mathrm{n}=2$ resonant magnetic perturbation field at jet. Nuclear Fusion, 52(5):054009, 2012. 